\title{
Stem cell therapy for cardiac repair: benefits and barriers
}

\section{Steven J. Joggerst and Antonis K. Hatzopoulos*}

Cardiovascular disease remains the leading cause of death worldwide. Acute ischaemic injury and chronic cardiomyopathies lead to permanent loss of cardiac tissue and ultimately heart failure. Current therapies aim largely to attenuate the pathological remodelling that occurs after injury and to reduce risk factors for cardiovascular disease. Studies in animal models indicate that transplantation of mesenchymal stem cells, bone-marrow-derived haematopoietic stem cells, skeletal myoblasts, or embryonic stem cells has the potential to improve the function of ventricular muscle after ischaemic injury. Clinical trials using primarily bone-marrow-derived cells and skeletal myoblasts have also produced some encouraging results. However, the current experimental evidence suggests that the benefits of cell therapy are modest, the generation of new cardiac tissue is low, and the predominant mechanisms of action of transplanted stem cells involve favourable paracrine effects on injured myocardium. Recent studies show that the adult heart possesses various pools of putative resident stem cells, raising the hope that these cells can be isolated for therapy or manipulated in vivo to improve the healing of cardiac muscle after injury. This article reviews the properties and potential of the various stem cell populations for cardiac repair and regeneration as well as the barriers that might lie ahead.

Cardiovascular disease is the leading cause of death worldwide. Of the almost 17 million people who die each year from cardiovascular causes, over 11 million die as a result of cardiac disease and 5.5 million deaths are related to stroke. Myocardial infarction carries a shortterm mortality rate of about $7 \%$ (with aggressive therapy), and congestive heart failure an even more distressing $20 \%$ one-year mortality (Ref. 1). Despite significant strides in therapy, thanks to newer treatment modalities and risk-reduction strategies, the global burden remains substantial (Refs 2, 3, 4, 5). This continued health problem has prompted research into new therapeutic approaches.

Stem cell therapy is a relatively new frontier in the battle against cardiovascular disease that has sparked intense research and criticism. With the

Vanderbilt University, Department of Medicine, Division of Cardiovascular Medicine, and Department of Cell \& Developmental Biology, Nashville, TN, USA.

*Corresponding author: Antonis K. Hatzopoulos, Vanderbilt University, Department of Medicine, Division of Cardiovascular Medicine, and Department of Cell \& Developmental Biology, MRB IV P425C, 2213 Garland Avenue, Nashville, TN 37232, USA. Tel: +1 615 936 5529; Fax: +1 615936 1872; E-mail: antonis.hatzopoulos@vanderbilt.edu

Accession information: doi:10.1017/S1462399409001124; Vol. 11; e2O; July 2009 (c) Cambridge University Press 2009. Re-use permitted under a Creative Commons Licence - by-nc-sa. 
discovery of various stem cell populations possessing cardiogenic potential, and the subsequent ability to isolate and expand these cells, the notion of a restorative therapy has begun to take shape. Although much knowledge has been gained through more than a decade of research, numerous barriers to true cardiac regeneration remain. In the pursuit of this endeavour, it has become apparent that we need to better understand the processes that lead to both damage and repair if we are to realise the true potential of stem cell therapy.

\section{Ischaemia and infarct}

Myocardial ischaemia, whether acute or chronic, begets a cascade of events leading to cellular injury or death with resultant scar formation and ultimately mechanical dysfunction, electrical uncoupling and loss of structural integrity (Ref. 6). Other than early restoration of blood flow, which engenders its own complications, the process is largely irreversible (Refs 7,8$)$. True regeneration is extremely limited.

Within seconds of an ischaemic insult, aerobic glycolysis ceases, leading to marked ATP depletion and lactic acid accumulation (Refs 9, 10). Early in the process, clinical reduction in myocardial contractility occurs secondary to the build up of various tissue metabolites that reduce the $\mathrm{Ca}^{2+}$ sensitivity of contractile myofilaments (Ref. 11). Continued oxygen deprivation leads to failure of the $\mathrm{Na}^{+} / \mathrm{K}^{+}$ATPase pump, an increase in intracellular solute, and subsequent swelling (Refs 7, 12). Accumulation of lactic acid reduces the cellular $\mathrm{pH}$, limiting the activity of essential enzymes and increasing the release of lysosomal products that lead to cellular breakdown. In addition, the failure of the $\mathrm{Ca}^{2+}$ pump leads to $\mathrm{Ca}^{2+}$ influx, with damaging effects on numerous intracellular components including ribosomal dissociation and mitochondrial membrane potential reduction, ultimately ending in apoptosis (Ref. 13). Cellular death signals macrophage and neutrophil infiltration, originally to the periphery and later to the centre of the infarct. As phagocytosis ensues, the necrotic tissue is removed and replaced with fibrovascular granulation tissue, leading to a decrease in the thickness of the muscle wall. As the process continues, neutrophils are replaced with myofibroblasts and subsequent deposition of collagen (predominantly type I and III). Finally, the cellularity is reduced, leaving only a dense collagenous scar (Ref. 6).

Scar formation is an essential aspect of rapid wound healing, especially in the injured myocardium, which is under constant wall stress. Without rapid wound healing, the ischaemic region would be subject to rupture, which is generally incompatible with life. Scar formation therefore offers protection from immediate danger by providing a rapid mechanical barrier (Ref. 14). However, scar tissue is largely acellular and lacks the normal biochemical properties of the host cells. This leads to electrical uncoupling, mechanical dysfunction, and loss of structural integrity, ultimately resulting in a dilated cardiomyopathy (Refs 15, 16). Limiting scar formation or even reversing the process could thus prove beneficial in maintaining the overall function of the organ.

To date, the mainstays in treatment of heart disease focus on reducing myocardial oxygen demand, increasing its supply and limiting the ischaemic burden in an effort to prevent scar formation and enhance myocardial function. However, once scar formation has occurred, a vicious cycle ensues, first with localised dysfunction and later with remodelling and dilation of the surrounding myocardium, leading to heart failure.

It is well known that following injury many species of amphibians and fish undergo complete regeneration (Refs 17, 18). Moreover, embryos respond differently than adults to tissue injury, with rapid, almost complete, regeneration and little scar formation (Ref. 19). This is believed to be a result of both the intrinsic function of embryonic fibroblasts as well as the external milieu surrounding the embryonic cells (Ref. 17). A better understanding of these intrinsic regenerative mechanisms may lead to novel potent therapies in the future.

The discovery of the proliferative capacity and plasticity of various stem cell populations has sparked much interest and debate regarding their use as a potential therapy. Over the past decade, several different stem cell types have been studied in an effort to find the best source for cardiac regeneration. Each stem cell population has its own advantages and complications (Table 1). Here, we examine the

Accession information: doi:10.1017/S1462399409001124; Vol. 11; e2O; July 2009 (c) Cambridge University Press 2009. Re-use permitted under a Creative Commons Licence - by-nc-sa. 


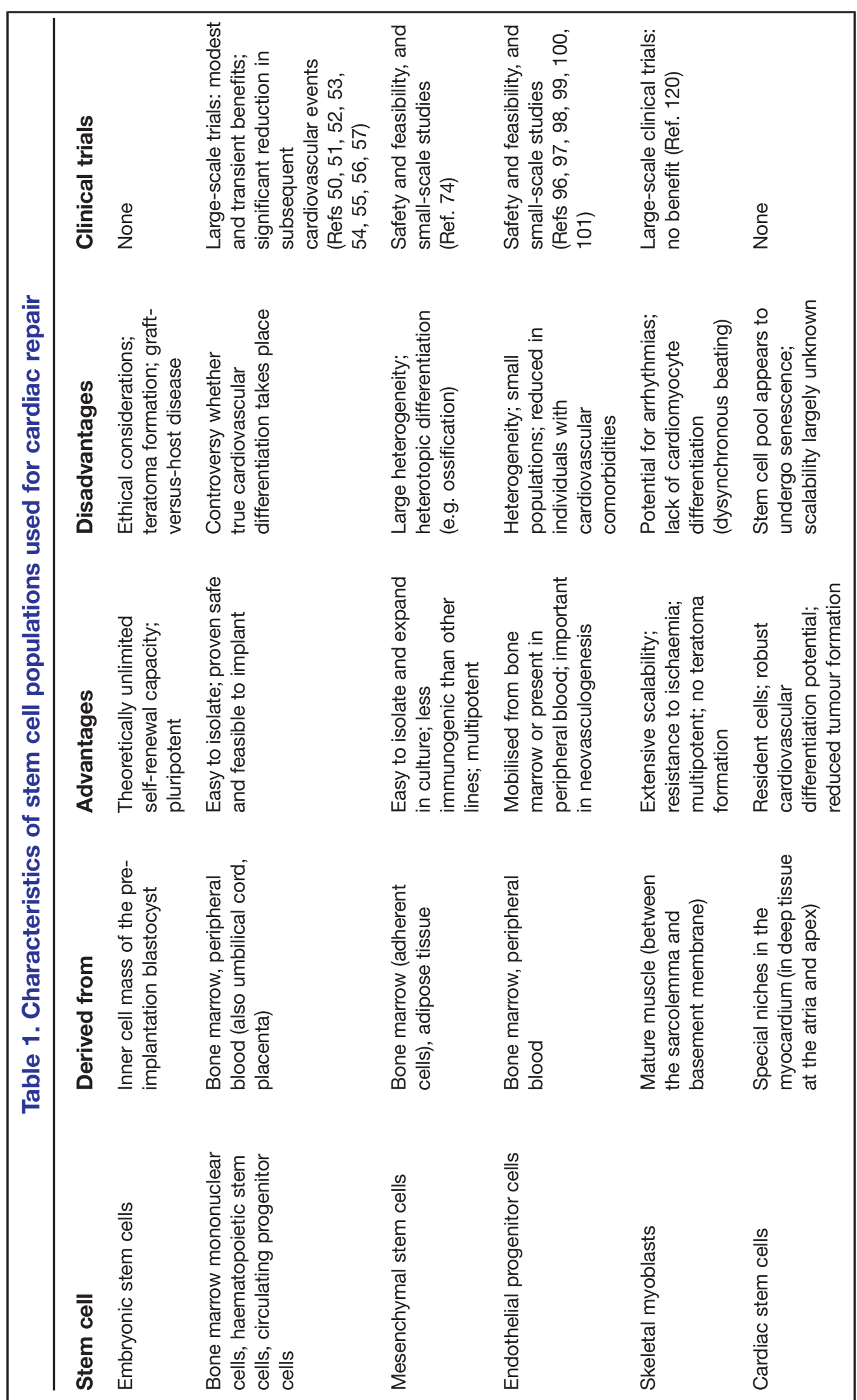


various stem cells utilised in animal models and clinical trials thus far, discussing briefly their benefits, disadvantages and evidence supporting their use.

\section{Embryonic stem cells}

Mouse and human embryonic stem cells (ESCs) can be removed from the inner mass of the blastocyst and expanded practically indefinitely in vitro (Refs 20, 21, 22). ESCs remain pluripotent in an undifferentiated state in culture; when allowed to differentiate, usually as embryoid bodies, ESCs are able to give rise to most somatic cell lineages (Refs 23, 24, 25). In this regard, their regenerative capacity is theoretically limitless. Furthermore, by culturing the embryoid bodies in various growth media, one can drive differentiation towards a desired cell type such as the cardiomyocyte (Refs 23, 24). These cells can then be implanted into the corresponding organ. This approach to repair cardiac tissue after injury has been tested in preclinical studies with encouraging results (Refs 26, 27, 28 , 29). In fact, of the various stem cell populations studied so far, perhaps the greatest capacity for cardiac cell differentiation and long-term cell survival has been seen in studies using ESCs (Ref. 30).

To date, no human trials have been attempted using ESCs for cardiac repair. There have been three main concerns regarding their use as a treatment modality. First, differentiating embryoid bodies contain cells from all three germ layers of ectoderm, mesoderm and endoderm, and therefore possess the capacity to differentiate along any or all of these lineages. This increases the likelihood of teratoma formation at the implantation site. Although these teratomas are believed to be largely benign in vivo, reasonable concerns have been raised because some cells have been found to express markers similar to those found in malignant tumors (Ref. 31). There is some evidence that the host tissues secrete factors that help drive the stem cells along a particular differentiation pattern (Refs 29, 32). Subsequently, there has been increased interest in culturing the embryoid bodies in specific media to promote differentiation to specific cell types (Ref. 33). These partially, or in some cases fully, differentiated cells can then be implanted, alleviating some of the risk of teratoma formation. Studies with these differentiated cells have shown increased engraftment and functional improvement (Refs 28, 30). While no long-term studies have been carried out to assess the real risk of teratoma formation, the theoretical concern remains an important obstacle.

The second issue regarding the use of ESCs pertains to immunity. Once thought to be uniquely immunoprivileged, increasing evidence has demonstrated that ESCs express specific human leukocyte antigen (HLA) subclasses (Ref. 34). This raises the worry of graft rejection and might necessitate immunosuppression. Steroid use without concomitant stem cell implantation has been known for some time to be harmful to ischaemic myocardium (Ref. 35). Not only does immunosuppression complicate the treatment with stem cells, but it may in fact undo any benefit derived from the addition of the stem cells to the ischaemic milieu. There is currently ongoing research to help limit the immunogenicity of the cells for allogeneic transplantation.

Finally, the origin of ESCs has raised considerable ethical concerns and led to heated debates among scientists and the wider public. The recent discovery that it is possible to generate ESC-like cells, called inducible pluripotent stem (iPS) cells, by reprogramming adult somatic cells with genes regulating ESC pluripotency may resolve the ethical and immunogenic issues associated with the use of ESCs (Refs 36, 37, 38).

\section{Bone-marrow-derived stem cells Bone marrow haematopoietic progenitor/ stem cells}

Bone marrow haematopoietic stem cells, or circulating peripheral blood progenitor cells, were shown to differentiate into cardiomyocytes in culture, making them of particular interest in the treatment of cardiac disease because they represent a wellcharacterised and ample source of progenitor cells (Refs 39, 40, 41, 42). A number of landmark studies showed significant improvement in cardiac function when bonemarrow-derived cells were implanted directly or mobilised from endogenous reservoirs. Some analyses not only showed improved ventricular function, but actually demonstrated 
regeneration of contracting cardiomyocytes and vascular beds (Refs 43, 44, 45, 46). However, other investigations found limited or no differentiation of bone marrow cells to cardiovascular cell types (Refs 47, 48), suggesting a beneficial effect independent of tissue regeneration (Ref. 49). Nevertheless, the improvements seen in ventricular function prompted a number of clinical trials using autologous bone marrow cells to treat heart failure patients or patients who had suffered a myocardial infarction. The clinical studies used circulating haematopoietic progenitor cells, or bone marrow mononuclear cells (MNCs), which also contain the small population of haematopoietic stem cells.

Early smaller studies were encouraging. However, larger, randomised, placebo-controlled and blinded studies have shown some mixed results (Refs 50, 51, 52, 53). The REPAIR-AMI trial (the largest of the randomised, placebo-controlled trials) was positive in that it not only demonstrated improved left ventricular function, but also showed a reduction in the combined clinical endpoint of death, myocardial infarction or revascularisation at one year (Ref. 54). The BOOST trial also showed improved left ventricular function early on compared with control patients, but by 18 months that difference had disappeared as control patients caught up with those who received cell therapy (Refs 55, 56). In contrast to the improved left ventricular function results of the REPAIR-AMI and BOOST trials, a double-blind, randomised controlled study, using autologous bone marrow MNCs in patients with myocardial infarction $24 \mathrm{~h}$ after successful percutaneous coronary intervention, showed no benefit in left ventricular ejection fraction, but a significant reduction in infarct size and improved regional left ventricular function (Ref. 53).

A recent meta-analysis of 18 randomised and nonrandomised trials involving 999 patients with acute myocardial infarction or chronic ischaemic cardiomyopathy found that transplantation of adult bone marrow cells improved left ventricular ejection fraction by $5.40 \%$, decreased infarct scar size by $5.49 \%$ and lowered left ventricular end-systolic volume by $4.80 \mathrm{ml}$ (Ref. 57).

It is possible that the apparently conflicting results among different trials are secondary to the cell preparation or the timing of the cell administration. There is clearly a need for further large-scale trials to assess the role of infused bone marrow cells in cardiac repair in order to improve their therapeutic efficacy.

\section{Mesenchymal stem cells}

Mesenchymal stem cells (MSCs) are a subset of stem cells that inhabit the stroma of bone marrow and can differentiate into osteoblasts, chondrocytes and adipocytes (Refs 58,59). They can be separated from haematopoietic cells by their ability to adhere to the culture dish (Ref. 60). MSCs can also be induced to differentiate in vitro into cardiomyocytes, which has stimulated a large number of animal and clinical studies to evaluate the efficacy of MSCs for cardiac repair and regeneration (Refs 61, 62, 63). MSCs are potentially advantageous as they are thought to be less immunogenic than other lines (Refs 64, 65). This alleviates the need for immunosuppression or autologous therapy.

Preclinical studies using transplantation of MSCs in post-infarct mice demonstrated improved left ventricular function and reduction in infarct size (Refs 62, 64, 65, 66, 67, 68, 69), and a decrease in mortality (Ref. 70). These improvements were seen despite small numbers of cells undergoing differentiation to cardiomyocytes (Refs 68, 71, 72, 73). A clinical study of MSCs in 69 postinfarct patients also demonstrated improved left ventricular function (Ref. 74).

Difficulties may arise, however, because of the broad differentiation capacity of MSCs. There remains significant heterogeneity among MSC populations and thus they are less predictable when implanted. Most notably, some studies found that implanted MSCs had differentiated into osteoblasts inside ventricular tissue (Refs 75, 76). This is an obvious cause for concern and needs to be addressed prior to fullscale therapy.

\section{Endothelial progenitor cells}

Another bone marrow cell type, the endothelial progenitor cell (EPC), has shown great promise as a potential therapy. Angiogenesis was once thought to occur solely though the proliferation of mature endothelial cells at sites of injury. This was challenged with the discovery that bone-marrow-derived EPCs home to sites of injury and incorporate into the microvasculature (Refs 77, 78). This revolutionised our understanding of vascular

Accession information: doi:10.1017/S1462399409001124; Vol. 11; e20; July 2009 (c) Cambridge University Press 2009. Re-use permitted under a Creative Commons Licence - by-nc-sa. 
growth and repair and became an intriguing concept for therapeutic manipulation.

Although there is some controversy regarding their true definition, EPCs can be identified by their ability to acquire endothelial cell characteristics in culture and in vivo. They express cell-surface makers such as cluster of differentiation molecule 133 (CD133), the vascular endothelial growth factor receptor 2 kinase (VEGFR-2; also known as KDR), CD34 and vascular endothelial cadherin (VE-cadherin). Of these, $\mathrm{CD} 34^{+}$and $\mathrm{CD} 133^{+}$ cells are the most widely recognised and utilised, although these markers are also shared by haematopoietic stem cells (Ref. 79). EPCs are mobilised from bone marrow in such injurious states as burns, myocardial infarction and cancer (Refs 80, 81, 82, 83). Furthermore, they have been shown to contribute anywhere from $5 \%$ to $25 \%$ of neovessel formation (Refs 84,85 ). Not only do EPCs aid in vasculogenesis, but there is also evidence that they can differentiate to cardiomyocytes (Ref. 86).

Subsequently, the search began to find ways to enhance their mobilisation or to directly incorporate them into the vasculature of injured tissues. Both VEGF and granulocyte colonystimulating factor (G-CSF) have been shown to increase EPC mobilisation from bone marrow (Refs 87, 88, 89). It should also be noted that statins (3-hydroxy-3-methylglutaryl coenzyme-A reductase inhibitors) have been shown to stimulate the mobilisation of EPCs from the bone marrow as well, pointing to yet another aspect of the ever-evolving understanding of the many therapeutic benefits of the drug (Refs 90, 91).

The first preclinical studies with implanted EPCs were hind-limb ischaemia experiments, which demonstrated significant improvement in blood flow recovery and limb salvage (Refs 77, 92, 93). Furthermore, injection of EPCs into infarcted myocardium improved left ventricular function and inhibited fibrosis (Refs 79, 94, 95). These results led to clinical experiments to assess safety and feasibility (Refs 96, 97, 98). The results of several small trials have shown trends toward improvement in left ventricular function with both acute and chronic ischaemia, without adverse effects (Refs 97, 99, 100, 101).

EPCs have already found a niche in the field of interventional cardiology. The earliest stents used were bare metal stents without drug coating. Although beneficial, these stents have an increased tendency to restenose (narrowing of the vessel via a localised inflammatory response) over time. Drug-eluting stents (impregnated with various chemicals that inhibit neointimal thickening) reduce the restenosis rate, but increase rates of in-stent thrombosis, a potentially fatal event. A newer technology for stents may be on the horizon. GENOUS stents are coated with anti-CD34 antibodies, which serve to trap circulating EPCs and augment the endothelialisation process in an effort to prevent restenosis (Ref. 102). They have already proven safe for implantation and ongoing studies will assess whether we are able to reduce the restenosis rate without the concern for in-stent thrombosis.

There are, however, barriers to the use of EPCs as therapeutic agents. First, is the heterogeneity of this cell population. EPCs circulating in the peripheral blood span the full range of differentiation from angioblasts to mature endothelial cells. This in part could explain differences in results from various studies. Second, the stem cell pool of EPCs is quite limited and only through ex vivo expansion can one attain appreciable numbers to surmount any significant injury or ischaemic event (Ref. 79). Last, the circulating pool of EPCs is reduced in patients with cardiac ischaemic disease comorbidities such as diabetes mellitus, hypertension and hypercholesterolaemia (Refs 103, 104). This is problematic as this cohort is essentially the very one that would need to be treated with EPCs - namely patients with coronary artery disease and other ischaemic risk factors. These challenges require further research to enhance the therapeutic efficiency of EPCs in ischaemic tissue.

\section{Skeletal myoblasts}

Skeletal myoblasts have been seen as an attractive source of stem cells and were among the earliest cell types considered for cardiac repair. Often called satellite cells, they are found beneath the basal membrane of muscle tissue where they lie dormant until stimulated to proliferate by muscle injury or disease (Ref. 105). These cells are further differentiated than ESCs and are thus less prone to teratoma formation. Furthermore, they can be harvested from the host, expanded in vitro, and autologously reimplanted, thus avoiding the need for immunosuppression (Ref. 106). Skeletal

Accession information: doi:10.1017/S1462399409001124; Vol. 11; e2O; July 2009 (c) Cambridge University Press 2009. Re-use permitted under a Creative Commons Licence - by-nc-sa. 
myoblasts are especially apt for cardiac repair as they are resistant to ischaemia, an inherent obstacle to the function of stem cells in injured myocardium (Ref. 107). Finally, skeletal myoblasts have the capacity to differentiate in vitro into nonmuscle cell types (Refs 108, 109). These properties prompted their consideration in cardiac repair.

Animal transplantation experiments in cardiac disease models were subsequently performed with encouraging results. Most of these studies showed improved left ventricular function and decreased remodelling possibly because the implanted cells form myotubules that are able to contract (an event possibly mediated by stretch receptors; Refs 106, 107, 110, 111, 112). Furthermore, the cells have been shown to decrease matrix breakdown both in the periinfarct area as well as remote myocardium, which likely contributes to reduced remodelling (Ref. 113).

However, skeletal myoblasts do not fully differentiate into cardiomyocytes in vivo after intramyocardial transplantation and the contracting myotubules do not operate in synchrony with the surrounding myocardium (Refs 112, 114). This is due at least in part to a lack of connexin activity and electrical coupling with the surrounding myocardial cells. However, regardless of the processes involved, the improvement in left ventricular function in animal models prompted a series of clinical investigations.

Early clinical studies were aimed at assessing the feasibility and safety of implantation (Refs 107, 115, 116, 117, 118, 119). These studies proved the therapy possible and showed that skeletal myoblasts survive in the human heart, although only marginal benefit was seen. Largerscale clinical trials were then undertaken to assess the benefit of myoblast therapy. The most notable to date was the MAGIC trial, which randomised patients to receive either stem cell injection or culture medium. Results from this trial have been disappointing in that no significant benefit was seen with stem cell implantation (Ref. 120). Further clinical studies are ongoing and may reveal differing results.

Several barriers still remain in the use of skeletal myoblasts. First, there has been considerable concern regarding the potential for arrhythmias (Refs 114, 121, 122). Early studies did report rare cases in human patients (Refs 117, 123). However, since then, there have been conflicting results and the data from more-recent large clinical trials did not record increased arrhythmic events in vivo after intracardial injection of skeletal myoblasts (Refs 120, 124). Animal experiments also showed that the electrical coupling of skeletal myoblasts to resident cardiomyocytes is increased when the skeletal cells are induced to overexpress connexin 43, indicating that there might be ways to overcome the arrhythmogenic obstacle (Refs 125, 126).

Another limitation is the relative paucity of engraftment of the injected cell population to the surrounding tissue. Cellular lethality of the order of $90 \%$ within the first few days has been demonstrated in mice (Ref. 127). Some studies in humans have shown similar cell death tolls (Ref. 107). The cells that survive are scarce. In addition, the engrafted cells differentiate into myotubules and not cardiomyocytes and therefore do not demonstrate a true regenerative therapy.

Finally, there is much variability and complexity involved in the use of skeletal myoblast populations. For example, female myoblasts demonstrate a higher proliferation potential than do male lines (Ref. 128). Moreover, although myoblasts are easy to harvest and expand in culture, the process is labour intensive and takes considerable time. This largely precludes autologous use in acute ischaemic events such as myocardial infarction.

\section{Cardiac stem cells}

The modest functional effects of transplanted progenitor cells from bone marrow and skeletal muscle in human studies stimulated further research into the natural regenerative mechanisms of the cardiac tissue. The heart has traditionally been viewed as a postmitotic organ because mature cardiomyocytes withdraw from the cell cycle and cease to proliferate. Interestingly, contradictory data began to accumulate as cardiomyocyte proliferation and cycling were found under certain pathological conditions - namely ischaemia and hypertension (Refs 129, 130, 131). This idea was further advanced with the discovery of male cardiomyocytes and endothelial cells in donor female cardiac tissue transplanted into a male

Accession information: doi:10.1017/S1462399409001124; Vol. 11; e2O; July 2009 (c) Cambridge University Press 2009. Re-use permitted under a Creative Commons Licence - by-nc-sa. 
recipient (Refs 132, 133). These findings raise the possibility that Y-chromosome positive, male cells migrated either from the recipient atrial stump or the bone marrow into the cardiac tissue and differentiated into functional cardiomyocytes. Moreover, estimates of the death rate levels of adult cardiomyocytes also led to the consideration of a pool of cardiac progenitor cells (Ref. 134). This evidence prompted a search to locate such resident cardiac cells. Subsequently, several different cell types were discovered in the adult heart with stem cell characteristics.

For example, a typical property of some stem cell populations is the cytoplasmic exclusion of vital dyes such as Hoechst 33342 and Rhodamine 123. The dye-negative cells have been called the side population (SP) cells. SP cells have been identified in various organs including bone marrow, skeletal muscle and adipose tissue (Ref. 135). Staining of dissociated cardiac tissue revealed that the heart also has a resident pool of SP cells (Refs 136, 137). Interestingly, isolated cardiac SP cells can differentiate to cardiomyocytes, suggesting that they represent cardiac progenitor cells (Refs 138, 139). SP cells are mobilised after cardiac injury (Ref. 140) but their regenerative potential is still unclear. One study documented differentiation of transplanted SP cells to cardiomyocytes, endothelial cells and smooth muscle cells (Ref. 139).

A second putative resident progenitor population comprises cells expressing the stem cell factor receptor c-Kit (also known as CD117), which are located in small clusters within the adult heart (Ref. 141). c-Kit ${ }^{+}$cells have regenerative potential after transplantation, giving rise to cardiomyocytes, endothelial cells and smooth muscle cells. c-Kit ${ }^{+}$cell transplantation after ischaemic injury leads to significant improvement in ventricular function (Refs 141, 142, 143, 144).

A third cell type in the heart with stem cell features consists of cells expressing the stem

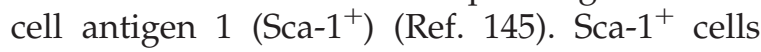
home to infarcted myocardium and differentiate to cardiomyocytes around the injury area (Ref. 145). The Sca- ${ }^{+}$cell subpopulation, which does not express CD31, was shown to differentiate into both cardiomyocytes and endothelial cells in culture (Ref. 146). Transplantation of Sca- $1^{+} \mathrm{CD} 31^{-}$cells in mice after myocardial infarction improved cardiac function and promoted new blood vessel formation (Ref. 146).
Finally, cardiac progenitors from mouse hearts were isolated by enzymatic digestion to obtain round cells that form so-called cardiospheres in suspension (Ref. 147). Cardiosphere-derived cells can differentiate to cardiomyocytes, endothelial cells and smooth muscle cells. An equivalent human cardiac stem cell population can be obtained via endomyocardial biopsy and subsequently grown in suspension as cardiospheres that exhibit remarkable proliferation and differentiation capacity (Refs 147, 148, 149). Once isolated, this cell population can be induced to differentiate into spontaneously beating aggregates of cardiomyocytes, which can then be implanted into injured myocardium at a later time (Refs 149, 150). The injection of cardiospherederived cells has shown some benefit in preclinical studies (Refs 148, 149, 150, 151). In much the same manner as the previous progenitor cell populations, the benefit appears to be largely by way of improved left ventricular function. There has been some regeneration seen in small numbers, but not enough to explain the functional improvement.

Cardiac stem cells (as well as stem cells from other tissues) appear to reside in specialised niches, which support the growth and maintenance of the stem cell pool (Refs 152, 153). Putative niches have been localised throughout the myocardium, concentrated in deep tissue at the atria and apex (Refs 141, 154). Recent evidence has also shown that there is a marked increase in the number and migration of such cells to the injury areas following an ischaemic insult (Ref. 145). Although the different cardiac stem cell pools are small relative to the mature resident cardiomyocytes, they are believed to be the source of new cells in normal organ homeostasis as well as in stressed myocardium (Ref. 155). At present, it is unclear if the various cardiac stem cells are distinct types or whether they represent different stages of a single cell lineage.

One seemingly contradictory aspect of endogenous cardiac stem cells is the apparent lack of regeneration seen in the chronic damage that occurs in ischaemic cardiomyopathy. It is puzzling why these pools of stem cells, which are induced to differentiate and migrate to sites of injury, are not able to reverse tissue losses. It is possible that the resident stem cell populations do not survive in the hypoxic

Accession information: doi:10.1017/S1462399409001124; Vol. 11; e20; July 2009 (c) Cambridge University Press 2009. Re-use permitted under a Creative Commons Licence - by-nc-sa. 
environment after an ischaemic insult and they undergo apoptosis along with mature myocardium. Furthermore, it appears that the cardiac stem cell pool diminishes with ageing, possibly contributing to the lack of efficacy of regeneration in elderly individuals (Ref. 155). Since it is largely the elderly who experience increased mortality from cardiomyopathies, it raises the need to enhance or rejuvenate this senescent stem cell population.

Favourable paracrine effects of stem cells As experimental evidence about the outcomes of stem cell therapy accumulated, a peculiar pattern began to emerge. Although many studies involving different stem cell populations and various administration modalities show significant benefit (often in the form of improved left ventricular function), there seems to be little differentiation of the infused stem cells into mature cardiovascular cell types. Moreover, few of the implanted cells persist for any appreciable length of time (Refs 127, 156). Also, the cardioprotective effects of stem cells are already evident $24 \mathrm{~h}$ after transplantation, a time frame that is too short for true regeneration (Ref. 157). These results have been recapitulated in many studies whereupon following a brief inhabitance in the ischaemic milieu the infused cells can no longer be found despite the persistent functional improvement of the myocardium (Ref. 158). Another peculiarity is a similar benefit has been derived using a wide range of stem cell populations. Finally, those studies that do demonstrate engraftment have shown numbers so small that it is hard to attribute the haemodynamic improvements to the incorporated cells.

The benefits witnessed therefore require further elucidation. If the implanted cells do not remain in the tissue and differentiate into functional cardiomyocytes in appreciable numbers, then how is this benefit derived? The hypothesis began to emerge that the stem cell populations exert a favourable paracrine effect on the injured myocardium, perhaps preventing apoptosis and promoting healing (Refs 156, 159).

Indeed, various studies showed that progenitor cells secret survival factors, which stimulate tissue recovery after ischaemic injury and minimise the infarct size (Refs 156, 157, 160, 161, 162). The beneficial effects have been thus far attributed to specific products of transplanted progenitor cells such as thymosin $\beta 4$, which promotes wound healing, or the Wnt antagonist SFRP-2 (secreted frizzled-related protein 2), which protects cardiomyocytes from hypoxia-induced apoptosis (Refs 163, 164, 165). In addition, based on the gene expression profiles of various stem cell types (Refs 156, 166), it is likely that stem-cell-secreted factors attenuate inflammation, decrease apoptosis, induce angiogenesis, recruit other stem cells and reduce the extent of fibrosis (Refs 156, 162, 167) (Fig. 1).

Taken together, the experimental evidence suggests that current benefits derived from stem cell therapy are at least in part secondary to a favourable paracrine effect of the stem cells acting on the host tissue. Whether or not the administration of isolated stem cell products or the physical presence of stem cells in the injury site is a more ideal form of therapy remains to be seen. However, it is apparent that in response to ischaemia many factors, acting in concert, work to limit damage and enhance repair. It is therefore possible that by providing the injured tissue with a functioning stem cell population, which can react to the internal milieu and respond with sustainable, targeted production of cardioprotective peptides, greater damage attenuation can be achieved than by simply infusing fixed quantities of specific agents. Perhaps the dynamic presence of a tissue repair biocatalyst is the most beneficial effect of stem cell implantation, which better equips injured tissue with the tools and blueprints to aid recovery and regeneration.

\section{Future directions}

Since the discovery of various resident stem cell populations and the subsequent ability to extract and culture them for therapeutic use, there has been a wealth of research into the potential of regenerating injured tissue. The current evidence suggests that stem cell therapy has great promise for attenuating remodelling and transforming inert scar into biochemically functional myocardium. However, the past decade has shown that translating the potential of stem cell therapy into actual practice is not easy, and many barriers would need to be overcome before this therapy attains its full potential.

Accession information: doi:10.1017/S1462399409001124; Vol. 11; e2O; July 2009 (c) Cambridge University Press 2009. Re-use permitted under a Creative Commons Licence - by-nc-sa. 
Figure 1. Putative paracrine effects of stem cells in ischaemic myocardium. Stem cells secrete factors that: promote survival of ischaemic cardiomyocytes and reduce apoptosis; induce angiogenesis, improving perfusion around the ischaemic area; modulate protease activity and scar formation; and produce factors that recruit circulating (pink) or resident (orange) progenitor cells. The improved disease environment attenuates inflammation and fibrosis, curtailing subsequent cardiac tissue remodelling (based on Refs 156, 162). On the figure, inflammation is depicted by a monocyte, macrophage and neutrophil; scar (or granulation) tissue is represented by myofibroblasts, macrophages and capillaries in a collagen matrix. Abbreviations: ANG, angiogenin; ANGPT, angiopoietin; CTGF, connective tissue growth factor; FGF, fibroblast growth factor; HGF, hepatocyte growth factor; IGF, insulin-like growth factor; IL, interleukin; LIF, leukaemia inhibitory factor; CCL2, chemokine (C-C motif) ligand 2 (also known as monocyte chemoattractant protein 1; MCP-1); MMP, matrix metalloproteinase; PDGF, platelet-derived growth factor; SCF, stem cell factor (c-Kit ligand); SDF, stromal-cell-derived factor; SFRP, secreted frizzled-related protein; T $\beta 4$, thymosin $\beta 4$; TGF, transforming growth factor; TIMP, tissue inhibitor of metalloproteinases; VEGF, vascular endothelial growth factor.

Despite these obstacles, the observed functional improvement with or without long-term engraftment of the stem cells has spurred continued animal and clinical studies along several different directions. First, there is ongoing research into ways to better enhance the recruitment, survival and long-term engraftment of implanted stem cells (Refs 168, 169). If true regeneration is to take place, then a sizeable percentage of the stem cells need to remain viable, differentiate into fully functional cardiomyocytes and incorporate into the resident tissue. Second, further analyses of stem cells that exhibit robust cardiac potential (i.e.

Accession information: doi:10.1017/S1462399409001124; Vol. 11; e20; July 2009 (C) Cambridge University Press 2009. Re-use permitted under a Creative Commons Licence - by-nc-sa. 
human ESCs and autologous iPS cells) are also needed to generate pure cell populations of cardiomyocytes with appropriate functional characteristics. Third, the interesting notion that stem cells exert their influence largely through paracrine activity has sparked research into how this effect is brought about. By gaining more understanding of the molecular interactions between donor stem cells and host tissue, we could discover ways to harness this effect. Finally, the discovery of various cardiac stem cell populations has renewed interest in the innate regenerative capacity of the human heart to enhance endogenous repair or mimic it with exogenous stem cell therapy. Although much more work needs to be done, stem cell therapies in conjunction with current treatment modalities may help to further reduce the mortality and improve the quality of life in cardiovascular disease patients.

\section{Acknowledgements and funding}

The authors thank the peer reviewers for their input and helpful advice. This work was supported by NIH grants HL083958 and HL087403 to A.K.H.

\section{References}

1 Assessment of the Safety and Efficacy of a New Thrombolytic Regimen (ASSENT)-3 Investigators et al. (2001) Efficacy and safety of tenecteplase in combination with enoxaparin, abciximab, or unfractionated heparin: the ASSENT-3 randomised trial in acute myocardial infarction. Lancet 358, 605-613

2 Manson, J.E. et al. (1992) The primary prevention of myocardial infarction. New England Journal of Medicine 326, 1406-1416

3 Braunwald, E. and Antman, E.M. (1997) Evidencebased coronary care. Annals of Internal Medicine 126, 551-553

4 Hunink, M.G. et al. (1997) The recent decline in mortality from coronary heat disease, 1980-1990. The effect of secular trends in risk factors and treatment. Journal of the American Medical Association 277, 535-542

5 McMurray, J.J. and Pfeffer, M.A. (2005) Heart failure. Lancet 365, 1877-1889

6 Frangogiannis, N.G. (2008) The immune system and cardiac repair. Pharmacological Research 58, 88-111

7 Jennings, R.B. et al. (1990) Development of cell injury in sustained acute ischemia. Circulation 82 (3 Suppl), II2-12
8 Yellon, D. and Hausenloy, D. (2007) Myocardial Reperfusion Injury. New England Journal of Medicine 357, 1121-1135

9 Bolli, R. and Marban, E. (1999) Molecular and cellular mechanisms of myocardial stunning. Physiological Reviews 79, 609-634

10 Kloner, R.A. et al. (1998) Medical and cellular implications of stunning, hibernation and preconditioning: an NHLBI workshop. Circulation 97, 1848-1867

11 Kumar, V. et al. (2005) Cellular adaptations, cell injury, and cell death. In Robbins and Cotran: Pathologic Basis of Disease ( $7^{\text {th }}$ edn) (Kumar, V. et al., eds), pp. 11-19, Elsevier, Philadelphia, USA

12 Newmeyer, D.D. and Ferguson-Miller, S. (2003)

Mitochondria: releasing power for life and unleashing the machineries of death. Cell 112,481-490

13 Trump, B.F. and Beezesky, I. (1998) The reactions of cells to lethal injury: oncosis and necrosis - the role of calcium. In When Cells Die - a Comprehensive Evaluation of Apoptosis and Programmed Cell Death (Lockshin, R.A., Zakeri, Z. and Tilly, J.L., eds), pp. 57-96, Wiley-Liss, New York, USA

14 Mutsaers, S.E. et al. (1997) Mechanisms of tissue repair: from wound healing to fibrosis.

International Journal of Biochemistry and Cell Biology 29, 5-17

15 Beltrami, C.A. et al. (1994) Structural basis of end-stage failure in ischemic cardiomyopathy in humans. Circulation 89, 151-163

16 Sun, Y. (2009) Myocardial repair/remodelling following infarction: roles of local factors. Cardiovascular Research 81, 482-490

17 Ferguson, M.W. and O'Kane, S. (2004) Scar-free healing: from embryonic mechanisms to adult therapeutic intervention. Philosophical Transactions of the Royal Society London B Biological Sciences 359, 839-850

18 Lepilina, A. et al. (2006) A dynamic epicardial injury response supports progenitor cell activity during zebrafish heart regeneration. Cell 127, 607-619

19 Mackool, R.J., Gittes, G.K. and Longaker, M.T. (1998) Scarless healing: the fetal wound. Clinics in Plastic Surgery 25, 357-361

20 Evans, M.J., and Kaufman, M.H. (1981) Establishment in culture of pluripotential cells from mouse embryos. Nature 292, 154-156

21 Martin, G.R. (1981) Isolation of a pluripotent cell line from early mouse embryos cultured in medium conditioned by teratocarcinoma stem cells.

Proceedings of the National Academy of Sciences of the United States of America 78, 7634-7638

Accession information: doi:10.1017/S1462399409001124; Vol. 11; e2O; July 2009

(c) Cambridge University Press 2009. Re-use permitted under a Creative Commons Licence - by-nc-sa. 
22 Thomson, J.A. et al. (1998) Embryonic stem cell lines derived from human blastocysts. Science 282, 1145-1147

23 Doetschman, T.C. et al. (1985) The in vitro development of blastocyst-derived embryonic stem cell lines: formation of visceral yolk sac, blood islands and myocardium. Journal of Embryology and Experimental Morphology 87, 27-45

24 Odorico, J.S., Kaufman, D.S. and Thomson, J.A. (2001) Multilineage differentiation from human embryonic stem cell lines. Stem Cells 19, 193-204

25 Murry, C.E. and Keller, G. (2008) Differentiation of embryonic stem cells to clinically relevant populations: lessons from embryonic development. Cell 132, 661-680

26 Min, J.Y. et al. (2002) Transplantation of embryonic stem cells improves cardiac function in post-infarcted rats. Journal of Applied Physiology 92, 288-296

27 Yang, Y. et al. (2002) VEGF enhances functional improvement of post-infarcted hearts by transplantation of ESC-differentiated cells. Journal of Applied Physiology 93, 1140-1151

28 Laflamme, M.A. et al. (2007) Cardiomyocytes derived from human embryonic stem cells in pro-survival factors enhance function of infracted rat hearts. Nature Biotechnology 25, 1015-1024

29 Behfar, A. et al. (2007) Cardiopoietic programming of embryonic stem cells for tumor-free heart repair. Journal of Experimental Medicine 204, 405-420

30 van Laake, L. et al. (2007) Human embryonic stem cell-derived cardiomyocytes survive and mature in the mouse heart and transiently improve function after myocardial infarction. Stem Cell Research 1, 9-24

31 Blum, B. and Benvenisty, N. (2008) The tumorigenicity of human embryonic stem cells. Advances in Cancer Research 100, 133-158

32 Kofidis, T. et al. (2005) Stimulation of paracrine pathways with growth factors enhances embryonic stem cell engraftment and host-specific differentiation in the heart after ischemic myocardial injury. Circulation 111, 2486-2493

33 Hao, J. et al. (2008) Dorsomorphin, a selective small molecule inhibitor of BMP signaling, promotes cardiomyogenesis in embryonic stem cells. PLoS ONE 3, e2904

34 Draper, J.S. et al. (2002) Surface antigens of human embryonic stem cells: changes upon differentiation in culture. Journal of Anatomy 200, 249-258

35 Silverman, H.S. and Pfeifer, M.P. (1987) Relation between use of anti-inflammatory agents and left ventricular free wall rupture during acute myocardial infarction. American Journal of Cardiology 59, 363-364

36 Okita, K., Ichisaka, T. and Yamanaka, S. (2007) Generation of germ-line competent induced pluripotent stem cells. Nature 448, 313-317

37 Takahashi, K. et al. (2007) Induction of pluripotent stem cells from adult human fibroblasts by defined factors. Cell 131, 861-872

38 Wernig, M. et al. (2007) In vitro reprogramming of fibroblasts into a pluripotent ES-cell-like state. Nature 448, 318-324

39 Yeh, E.T. et al. (2003) Transdifferentiation of human peripheral blood CD34-enriched cell population into cardiomyocytes, endothelial cells, and smooth muscle cells in vivo. Circulation 108, 2070-2073

40 Belema Bedada, F. et al. (2005) Activation of myogenic differentiation pathways in adult bone marrow-derived stem cells. Molecular and Cellular Biology 25, 9509-9519

41 Koyanagi, M. et al. (2007) Notch signaling contributes to the expression of cardiac makers in human circulating progenitor cells. Circulation Research 101, 1139-1145

42 Flaherty, M.P. et al. (2008) Noncanonical Wnt11 signaling is sufficient to induce cardiomyogenic differentiation in unfractionated bone marrow mononuclear cells. Circulation 117, 2241-2252

43 Orlic, D. et al. (2001) Bone marrow cells regenerate infarcted myocardium. Nature 410, 701-705

44 Orlic, D. et al. (2001) Mobilized bone marrow cells repair the infarcted heart, improving function and survival. Proceedings of the National Academy of Sciences of the United States of America 98, 10344-10349

45 Kajstura, J. et al. (2005) Bone marrow cells differentiate in cardiac cell lineages after infarction independently of cell fusion. Circulation Research 96, 127-137

46 Rota, M. et al. (2007) Bone marrow cells adopt the cardiomyogenic fate in vivo. Proceedings of the National Academy of Sciences of the United States of America 104, 17783-17788

47 Balsam, L.B. et al. (2004) Haematopoietic stem cells adopt mature haematopoietic fates in ischaemic myocardium. Nature 428, 668-673

48 Murry, C.E. et al. (2004) Haematopoietic stem cells do not transdifferentiate into cardiac myocytes in myocardial infarcts. Nature 428, 664-668

49 Kamihata, H. et al. (2001) Implantation of bone marrow mononuclear cells into ischemic myocardium enhances collateral perfusion and

Accession information: doi:10.1017/S1462399409001124; Vol. 11; e2O; July 2009

(c) Cambridge University Press 2009. Re-use permitted under a Creative Commons Licence - by-nc-sa. 
regional function via side supply of angioblasts, angiogenic ligands, and cytokines. Circulation 104, 1046-1052

50 Assmus, B. et al. (2002) Transplantation of progenitor cells and regeneration enhancement in acute myocardial infarction (TOPCARE-AMI). Circulation 106, 3009-3017

51 Lunde, K. et al. (2005) Autologous stem cell transplantation in acute myocardial infarction: the ASTAMI randomized controlled trial. Intracoronary transplantation of autologous mononuclear bone marrow cells, study design and safety aspects. Scandinavian Cardiovascular Journal 39, 150-158

52 Schachinger, V. et al. (2006) Intracoronary bone marrow-derived progenitor cells in acute myocardial infarction. New England Journal of Medicine 355, 1210-1221

53 Janssens, S. et al. (2006) Autologous bone marrow-derived stem-cell transfer in patients with ST-segment elevation myocardial infarction: double-blind, randomised controlled trial. Lancet 367, 113-121

54 Schachinger, V. et al. (2006) Improved clinical outcome after intracoronary administration of bone-marrow-derived progenitor cells I acute myocardial infarction: final 1-year results of the REPAIR-AMI trial. European Heart Journal 23, 2775-2783

55 Wollert, K.C. et al. (2004) Intracoronary autologous bone marrow cell transfer after myocardial infarction: the BOOST randomized controlled clinical trial. Lancet 364, 141-148

56 Meyer, G.P. et al. (2006) Intracoronary bone marrow cell transfer after myocardial infarction: eighteen months' follow-up data from the randomized, controlled BOOST (BOne marrOw transfer to enhance ST-elevation infarct regeneration) trial. Circulation 113, 1287-1294

57 Abdel-Latif, A. et al. (2007) Adult bone marrowderived cells for cardiac repair: a systematic review and meta-analysis. Archives of Internal Medicine 167, 989-997

58 Pittenger, M.F. et al. (1999) Multilineage potential of adult human mesenchymal stem cells.

Science 284, 143-147

59 Jiang, Y. et al. (2002) Pluripotency of mesenchymal stem cells derived from adult marrow. Nature $418,41-49$

60 Alhadlaq, A. and Mao, J.J. (2004) Mesenchymal stem cells: isolation and therapeutics. Stem Cells and Development 13, 436-448
61 Makino, S. et al. (1999) Cardiomyocytes can be generated from marrow stromal cells in vitro. Journal of Clinical Investigation 103, 697-705

62 Tomita, S. et al. (1999) Autologous transplantation of bone marrow cells improves damaged heart function. Circulation 100 (19 Suppl), II247-256

63 Shiota, M. et al. (2007) Isolation and characterization of bone marrow-derived mesenchymal progenitor cells with myogenic and neuronal properties. Experimental Cell Research 313, 1008-1023

64 Amado, L.C. et al. (2005) Cardiac repair with intramyocardial injection of allogeneic mesenchymal stem cells after myocardial infarction. Proceedings of the National Academy of Sciences of the United States of America 102, 11474-11479

65 Dai, W. et al. (2005) Allogeneic mesenchymal stem cell transplantation in postinfarcted rat myocardium: short- and long-term effects. Circulation 112, 214-223

66 Toma, C. et al. (2002) Human mesenchyman stem cells differentiate to a cardiomyocyte phenotype in the adult murine heart. Circulation 105, 93-98

67 Kudo, M. et al. (2003) Implantation of bone marrow stem cells reduces the infarction and fibrosis in ischemic mouse heart. Journal of Molecular and Cellular Cardiology 35, 1113-1119

68 Silva, G.V. et al. (2005) Mesenchymal stem cells differentiate into an endothelial phenotype, enhance vascular density, and improve heart function in a canine chronic ischemia model. Circulation 111, 150-156

69 Grauss, R.W. et al. (2008) Forced myocardin expression enhances the therapeutic effect of human mesenchymal stem cells after transplantation in ischemic mouse hearts. Stem Cells 26, 1083-1093

70 Miyahara, Y. et al. (2006) Monolayered mesenchymal stem cells repair scarred myocardium after myocardial infarction. Nature Medicine 12, 459-465

71 Fazel, S. et al. (2005) Cell transplantation preserves cardiac function after infarction by infarct stabilization: augmentation by stem cell factor. The Journal of Thoracic and Cardiovascular Surgery 130, 1310.el-1310.e10

72 Noiseux, N. et al. (2006) Mesenchymal stem cells overexpressing Akt dramatically repair infarcted myocardium and improve cardiac function despite

Accession information: doi:10.1017/S1462399409001124; Vol. 11; e20; July 2009

(c) Cambridge University Press 2009. Re-use permitted under a Creative Commons Licence - by-nc-sa. 
infrequent cellular fusion or differentiation. Molecular Therapy 14, 840-850

73 Rose, R.A. et al. (2008) Bone marrow-derived mesenchymal stromal cells express cardiac-specific markers, retain the stromal phenotype, and do not become functional cardiomyocytes in vitro. Stem Cells 26, 2884-2892

74 Chen, S.L. et al. (2004) Effect on left ventricular function of intracoronary transplantation of autologous bone marrow mesenchymal stem cells in patients with acute myocardial infarction. American Journal of Cardiology 94, 92-95

75 Yoon, Y.S. et al. (2004) Unexpected severe calcification after transplantation of bone marrow cells in acute myocardial infarction. Circulation 109, 3154-3157

76 Breitbach, M. et al. (2007) Potential risks of bone marrow cell transplantation into infarcted hearts. Blood 110, 1362-1369

77 Asahara, T. et al. (1997) Isolation of putative progenitor endothelial cells for angiogenesis. Science 275, 964-967

78 Shi, Q. et al. (1998) Evidence for circulating bone-marrow derived endothelial cells. Blood 92, 362-367

79 Jujo, K. et al. (2008) Endothelial progenitor cells in neovasculaization of infracted myocardium. Journal of Molecular and Cellular Cardiology 45, 530-544

80 Gill, M. et al. (2001) Vascular trauma induces rapid but transient mobilization of VEGFR2+ AC133+ endothelial precursor cells. Circulation Research 88, 167-174

81 Shintani, S. et al. (2001) Mobilization of endothelial progenitor cells in patients with acute myocardial infarction. Circulation 103, 2776-2779

82 Lamparter, M. and Hatzopoulos, A.K. (2007) The biology of embryonic and adult endothelial progenitor cells. In Therapeutic Neovascularization - Quo Vadis? (Deindl, E. and Kupatt, C., eds), pp. 197-213, Springer SBN, Dordrecht, The Netherlands

83 Lamparter, M. and Hatzopoulos, A.K. (2007) The role of endothelial progenitor cells in tumor angiogenesis. In Angiogenesis: Basic Science and Clinical Applications (Maragoudakis, M.E. and Papadimitriou, E., eds) pp. 17-30, Transworld Research Network, Kerala, India

84 Murayama, T. et al. (2002) Determination of bone marrow-derived endothelial progenitor cell significance in angiogenic growth factor-inducted neovascularization in vivo. Experimental Hematology 30, 967-972

85 Crosby, J.R. et al. (2000) Endothelial cells of hematopoietic origin make a significant contribution to adult blood vessel formation. Circulation Research 87, 728-730

86 Iwasaki, H. et al. (2006) Dose-dependent contribution of CD34-positive cell transplantation to concurrent vasculogenesis and cardiomyogenesis for functional regenerative recovery after myocardial infarction. Circulation 113, 1311-1325

87 Takahashi, T. et al. (1999) Ischemia- and cytokineinduced mobilization of bone marrow-derived endothelial progenitor cells for neovascularization. Nature Medicine 5, 434-438

88 Kalka, C. et al. (2000) VEGF gene transfer mobilizes endothelial progenitor cells in patients with inoperable coronary disease. The Annals of Thoracic Surgery 70, 829-834

89 Fukuhara, S. et al. (2004) G-CSF promotes bone marrow cells to migrate into infarcted mice heart and differentiate into cardiomyocytes. Cell Transplant 13, 741-748

90 Kureishi, Y. et al. (2000) The HMG-CoA reductase inhibitor simvastatin activates the protein kinase Akt and promotes angiogenesis in normocholesterolemic animals. Nature Medicine 6, 1004-1010

91 Llevadot, J. et al. (2001) HMG-CoA reductase inhibitor mobilizes bone marrow-derived endothelial progenitor cells. Journal of Clinical Investigation 108, 399-405

92 Kalka, C. et al. (2000) Transplantation of ex vivo expanded endotheilial progenitor cells for therapeutic neovascularization. Proceedings of the National Academy of Sciences of the United States of America 97, 3422-3427

93 Murohara, T. (2001) Therapeutic vasculogenesis using human cord blood-derived endothelial progenitors. Trends in Cardiovascular Medicine 11, 303-307

94 Kocher, A.A. et al. (2001) Neovascularization of ischemic myocardium by human bone-marrowderived angioblasts prevents cardiomyocyte apoptosis, reduces remodeling and improves cardiac function. Nature Medicine 7, 430-436

95 Kawamoto, A. et al. (2003) Intramyocardial transplantation of autologous endothelial progenitor cells for therapeutic neovascularization of myocardial ischemia. Circulation 107, 461-468

96 Stamm, C. et al. (2007) Intramyocardial delivery of CD133+ bone marrow cells and coronary artery 
bypass grafting for chronic ischemic heart disease: safety and efficacy studies. Journal of Thoracic and Cardiovascular Surgery 133, 717-725

97 Losordo, D.W. et al. (2007) Intramyocardial transplantation of autologous CD34+ stem cells for intractable angina: a phase I/IIa double-blind, randomized controlled trial. Circulation 115, 3165-3172

98 Bartunek, J. et al. (2005) Intracoronary injection of CD133-positive enriched bone marrow progenitor cells promotes cardiac recovery after recent myocardial infarction: feasibility and safety. Circulation 112 (9 Suppl), I178-183

99 Erbs, S. et al. (2005) Transplantation of blood-derived progenitor cells after recanalization of chronic coronary artery occlusion: first randomized and placebo-controlled study. Circulation Research 97, 756-762

100 Stamm, C. et al. (2003) Autologous bone-marrow stem-cell transplantation for myocardial regeneration. Lancet 361, 45-46

101 Klein, H.M. et al. (2007) Intramyocardial implantation of CD133+ stem cells improved cardiac function without bypass surgery. Heart Surgery Forum 10, E66-69

102 Aoki, J. et al. (2005) Endothelial progenitor cell capture by stents coated with antibody against CD34: the HEALING-FIM (Healthy Endothelial Accelerated Lining Inhibits Neointimal GrowthFirst In Man) Registry. Journal of American College of Cardiology 45, 1574-1579

103 Vasa, M. et al. (2001) Number and migratory activity of circulating endothelial progenitor cells inversely correlate with risk factors for coronary artery disease. Circulation Research 89, E1-7

104 Imanishi, T. et al. (2005) Endothelial progenitor cell senescence is accelerated in both experimental hypertensive rats and patients with essential hypertension. Journal of Hypertension 23, 1831-1837

105 Buckingham, M. and Montarras, D. (2008) Skeletal muscle stem cells. Current Opinion in Genetics and Development 18, 330-336

106 Murry, C.E. et al. (1996) Skeletal myoblast transplantation for repair of myocardial necrosis. Journal of Clinical Investigation 98, 2512-2523

107 Pagani, F.D. et al. (2003) Autologous skeletal myoblasts transplanted to ischemia-damaged myocardium in humans. Histological analysis of cell survival and differentiation. Journal of American College of Cardiology 41, 879-888
108 Asakura, A., Komaki, M. and Rudnicki, M. (2001) Muscle satellite cells are multipotential stem cells that exhibit myogenic, osteogenic, and adipogenic differentiation. Differentiation 68, 245-253

109 Arsic, N. et al. (2008) Muscle-derived stem cells isolated as non-adherent population give rise to cardiac, skeletal muscle and neural lineages.

Experimental Cell Research 314, 1266-1280

110 Taylor, D.A. et al. (1998) Regenerating functional myocardium: improved performance after skeletal myoblast transplantation. Nature Medicine 4, 929-933

111 Ghostine, S. et al. (2002) Long-term efficacy of myoblast transplantation on regional structure and function after myocardial infarction. Circulation 106 (12 Suppl 1), I131-136

112 Leobon, B. et al. (2003) Myoblasts transplanted into rat infracted myocardium are functionally isolated from their host. Proceedings of the National Academy of Sciences of the United States of America 100, 7808-7811

113 Farahmand, P. et al. (2008) Skeletal myoblasts preserve remote matrix architecture and global function when implanted early or late after coronary ligation into infracted or remote myocardium. Circulation 118 (14 Suppl), S130-137

114 Reinecke, H., Poppa, V. and Murry, C.E. (2002) Skeletal muscle stem cells do not transdifferentiate into cardiomyoctyes after cardiac grafting. Journal of Molecular and Cellular Cardiology 34, 241-249

115 Menasche, P. et al. (2003) Autologous skeletal myoblast transplantation for severe postinfarction left ventricular dysfunction. Journal of American College of Cardiology 41, 1078-1083

116 Herreros, J. et al. (2003) Autologous intramyocardial injection of cultured skeletal muscle-derived stem cells in patients with non-acute myocardial infarction. European Heart Journal 24, 2012-2020

117 Smits, P.C. et al. (2003) Catheter-based intramyocardial injection of autologous skeletal myoblasts as primary treatment of ischemic heart failure: clinical experience with six-month follow-up. Journal of the American College of Cardiology 42, 2063-2069

118 Dib, N. et al. (2005) Feasibility and safety of autologous myoblast transplantation in patients with ischemic cardiomyopathy. Cell Transplant 14, 11-19

119 Siminiak, T. et al. (2005) Percutaneous trans-coronary-venous transplantation of autologous skeletal myoblasts in the treatment of

$$
\text { American College of Cardiology 41, 879-888 }
$$

Accession information: doi:10.1017/S1462399409001124; Vol. 11; e20; July 2009 (c) Cambridge University Press 2009. Re-use permitted under a Creative Commons Licence - by-nc-sa. 
post-infarction myocardial contractility impairment: the POZNAN trial. European Heart Journal 26, 1188-1195

120 Menasche, P. et al. (2008) The Myoblast Autologous Grafting in Ischemic Cardiomyopathy (MAGIC) trial: first randomized placebo-controlled study of myoblast transplantation. Circulation 117, 1189-2000

121 Fouts, K. et al. (2006) Electrophysiological consequence of skeletal myoblast transplantation in normal and infarcted canine myocardium. Heart Rhythm 3, 452-461

122 Fernandes, S. et al. (2006) Autologous myoblast transplantation after myocardial infarction increases the inducibility of ventricular arrhythmias. Cardiovascular Research 69, 348-358

123 Itabashi, Y. et al. (2005) Analysis of the electrophysiological properties and arrhythmias in directly contacted skeletal and cardiac muscle cell sheets. Cardiovascular Research 6, 561-570

124 Hagege, A.A. et al. (2003) Viability and differentiation of autologous skeletal myoblast grafts in ischaemic cardiomyopathy. Lancet 361, 491-492

125 Abraham, M.R. et al. (2005) Antiarrhythmic engineering of skeletal myoblasts for cardiac transplantation. Circulation Research 97, 159-167

126 Roell, W. et al. (2007) Engraftment of connexin 43-expressing cells prevents post-infarct arrhythmia. Nature 450, 819-824

127 Suzuki, K. et al. (2004) Dynamics and mediators of acute graft attrition after myoblast transplantation to the heart. FASEB Journal 18, 1153-1155

128 Deasy, B.M. et al. (2007) A role for cell sex in stem cell-mediated skeletal muscle regeneration: female cells have higher muscle regeneration efficiency. Journal of Cell Biology 177, 73-86

129 Anvesa, P. et al. (1990) Hypertensive cardiomyopathy: myocyte nuclei hyperplasia in the mammalian at heart. Journal of Clinical Investigation 85, 994-997

130 Kajstura, J. et al. (1998) Myocyte proliferation in end-stage cardiac failure in humans. Proceedings of the National Academy of Sciences of the United States of America 95, 8801-8805

131 Beltrami, A.P. et al. (2001) Evidence that human cardiac myocytes divide after myocardial infarction. New England Journal of Medicine 344, $1750-1757$

132 Quaini, F. et al. (2002) Chimerism of the transplanted heart. New England Journal of Medicine 346, 5-15
133 Bayes-Genis, A. et al. (2007) Chimerism and microchimerism of the human heart: evidence for cardiac regeneration. Nature Clinical Practice Cardiovascular Medicine 4 (Suppl 1), S40-45

134 Ellison, G.M. et al. (2007) Myocyte death and renewal: modern concepts of cardiac cellular homeostasis. Nature Clinical Practice

Cardiovascular Medicine 4 (Suppl 1), S52-59

135 Challen, G.A. and Little, M.H. (2006) A side order of stem cells: the SP phenotype. Stem Cells 24, 3-12

136 Hierlihy, A.M. et al. (2002) The post-natal heart contains a myocardial stem cell population. FEBS Letters 530, 239-243

137 Martin, C.M. et al. (2004) Persistent expression of the ATP-binding cassette transporter, Abcg2, identifies cardiac SP cells in the developing and adult heart. Developmental Biology 265, 262-275

138 Pfister, O. et al. (2005) CD31- but not CD31+ cardiac side population cells exhibit functional cardiomyogenic differentiation. Circulation Research 97, 52-61

139 Oyama, T. et al. (2007) Cardiac side population cells have a potential to migrate and differentiate into cardiomyocytes in vitro and in vivo. Journal of Cell Biology 176, 329-341

140 Mouquet, F. et al. (2005) Restoration of cardiac progenitor cells after myocardial infarction by self-proliferation and selective homing of bone marrow-derived stem cells. Circulation Research 97, 1090-1092

141 Beltrami, A.P. et al. (2003) Adult cardiac stem cells are multipotent and support myocardial regeneration. Cell 114, 763-776

142 Dawn, B. et al. (2005) Cardiac stem cells delivered intravascularly traverse the vessel barrier, regenerate infarcted myocardium, and improve cardiac function. Proceedings of the National Academy of Sciences of the United States of America 102, 3766-3771

143 Bearzi, C. et al. (2007) Human cardiac stem cells. Proceedings of the National Academy of Sciences of the United States of America 104, 14068-14073

144 Rota, M. et al. (2008) Local activation or implantation of cardiac progenitor cells rescues scarred infarcted myocardium improving cardiac function. Circulation Research 103, 107-116

145 Oh, H. et al. (2003) Cardiac progenitor cells from adult myocardium: homing, differentiation, and fusion after infarction. Proceedings of the 
National Academy of Sciences of the United States of America 100, 12313-12318

146 Wang, X. et al. (2006) The role of the sca-1/CD31cardiac progenitor cell population in postinfarction left ventricular remodeling. Stem Cells 24, 1779-1788

147 Messina, E. et al. (2004) Isolation and expansion of adult cardiac stem cells from human and murine heart. Circulation Research 95, 911-921

148 Smith, R. et al. (2007) Regenerative potential of cardiosphere-derived cells expanded from percutaneous endomyocardial biopsy specimens. Circulation 115, 896-908

149 van Vliet, P. et al. (2008) Progenitor cells isolated from the human heart: a potential cell source for regenerative therapy. Netherlands Heart Journal 16, 163-169

150 Takehara, N. et al. (2008) Controlled delivery of basic fibroblast growth factor promotes human cardiosphere-derived cell engraftment to enhance cardiac repair for chronic myocardial infarction. Journal of American College of Cardiology 52, 1858-1865

151 Linke, A. et al. (2005) Cardiac stem cells in the dog heat regenerate infarcted myocardium improving cardiac performance. Proceedings of the National Academy of Sciences of the United States of America 102, 8966-8971

152 Fuchs, E., Tumbar, T. and Guasch, G. (2004) Socializing with the neighbors: stem cells and their niche. Cell 116, 769-778

153 Moore, K.A. and Lemischka, I.R. (2006) Stem cells and their niches. Science 311, 1880-1885

154 Urbanek, K. et al. (2005) Myocardial regeneration by activation of multipotent cardiac stem cells in ischemic heart failure. Proceedings of the National Academy of Sciences of the United States of America 102, 8692-8697

155 Torella, D. et al. (2006) Resident human cardiac stem cells: role in cardiac cellular homeostasis and potential for myocardial regeneration. Nature Clinical Practice Cardiovascular Medicine 3 (Suppl 1), S8-13

156 Kupatt, C. et al. (2005) Embryonic endothelial progenitor cells expressing a broad range of proangiogenic and remodeling factors enhance vascularizatin and tissue recovery in acute and chronic ischemia. FASEB Journal 19, 1576-1578

157 Kupatt, C. et al. (2005) Retroinfusion of embryonic endothelial progenitor cells attenuates ischemia-reperfusion injury in pig: role of PI3-Kinase/AKT. Circulation 112 (9 Suppl), I117-122

158 Laflamme, M.A. and Murry, C.E. (2005)

Regenerating the heart. Nature Biotechnology 23, 845-856

159 Heil, M. et al. (2004) A different outlook on the role of bone marrow stem cells in vascular growth, bone marrow delivers the software not hardware.

Circulation Research 94, 573-574

160 Gnecchi, M. et al. (2005) Paracrine action accounts for marked protection of ischemic heart by Akt-modified mesenchymal stem cells. Nature Medicine 11, 367-368

161 Uemura, R. et al. (2006) Bone marrow stem cells prevent left ventricular remodeling of ischemic heart through paracrine signaling. Circulation Research 98, 1414-1421

162 Gnecchi, M. et al. (2008) Paracrine mechanisms in adult stem cell signaling and therapy.

Circulation Research 103, 1204-1219

163 Hinkel, R. et al. (2008) Thymosin $\beta 4$ is an essential paracrine factor of embryonic endothelial progenitor cell mediated cardioprotection. Circulation 117, 2232-2240

164 Mirotsou, M. et al. (2007) Secreted frizzled related protein 2 (Sfrp2) is the key Akt-mesenchymal stem cell-released paracrine factor mediating myocardial survival and repair. Proceedings of the National Academy of Sciences of the United States of America 104, 1643-1648

165 Alfaro, M.P. et al. (2008) The Wnt modulator sFRP2 enhances mesenchymal stem cell engraftment, granulation tissue formation and myocardial repair. Proceedings of the National Academy of Sciences of the United States of America 105, 18366-18371

166 Caplan, A.I. and Dennis, J.E. (2006) Mesenchymal stem cells as trophic mediators. Journal of Cellular Biochemistry 98, 1076-1084

167 Haider, H.K.H. et al. (2008) IGF-1 overexpressing mesenchymal stem cells accelerate bone marrow stem cell mobilization via paracrine activation of SDF-1alpha/CXCR4 signaling to promote myocardial repair. Circulation Research 103, $1300-1308$

168 Ryzhov, S. et al. (2008) Adenosine receptormediated adhesion of endothelial progenitors to cardiac microvascular endothelial cells. Circulation Research 102, 356-363

169 Zhang, G. et al. (2008) Enhancing efficacy of stem cell transplantation to the heart with PEGylated fibrin biomatrix. Tissue Engineering Part A 14, $1025-1036$

Accession information: doi:10.1017/S1462399409001124; Vol. 11; e2O; July 2009 (c) Cambridge University Press 2009. Re-use permitted under a Creative Commons Licence - by-nc-sa. 


\section{Further reading, resources and contacts}

\section{Publications}

Leri, A., Kajstura, J. and Anversa, P. (2005) Cardiac stem cells and mechanisms of myocardial regeneration. Physiological Reviews 85, 1373-1416

A comprehensive overview of theoretical and practical aspects of cardiac regeneration.

Menasche, P. (2008) Skeletal myoblasts and cardiac repair. Journal of Molecular and Cellular Cardiology 45, 545-553

A frank discussion regarding the past and future of skeletal myoblasts for cardiac repair.

Bergmann, O. et al. (2009) Evidence for cardiomyocyte renewal in humans. Science 324, 98-102

Measuring ${ }^{14} \mathrm{C}$ (which did not exist in the atmosphere prior to nuclear testing) incorporation into human cardiomyocytic DNA, this interesting study concludes that the adult human heart regenerates with a slow, age-dependent, pace.

Burt, R.K. et al. (2008) Clinical applications of blood-derived and marrow-derived stem cells for nonmalignant diseases. Journal of the American Medical Association 299, 925-936

A detailed summary of clinical trials using blood- and bone-marrow-derived stem cells to treat nonmalignant, nonhaematological indications.

Chien, K.R., Domian, I.J. and Parker, K.K. (2008) Cardiogenesis and the complex biology of regenerative cardiovascular medicine. Science 322, 1494-1497

A fascinating review focusing on the regenerative potential of the embryonic cardiac progenitor cell population delineated by expression of the transcription factor islet-1.

Dimmeler, S. and Zeiher, A.M. (2008) Cell therapy of acute myocardial infarction: open questions. Cardiology $113,155-160$

A concise assessment of important issues in the clinical application of stem cells to treat cadiac disease.

Reffelmann, T., Könemann, S. and Kloner, R.A. (2009) Promise of blood- and bone marrow-derived stem cell transplantation for functional cardiac repair: putting it in perspective with existing therapy. Journal of the American College of Cardiology 53, 305-308

An intriguing analysis showing that functional improvements achieved by cell transplantation are comparable with established therapeutic strategies.

Reinecke, H. et al. (2008) Cardiogenic differentiation and transdifferentiation of progenitor cells. Circulation Research 103, 1058-1071

A stimulating discussion about the cardiogenic potential of various stem cell types.

Segers, V.F. and Lee, R.T. (2008) Stem-cell therapy for cardiac disease. Nature 451, 937-942

A concise, well-illustrated summary about the outcome of stem cell therapies for cardiac disease.

Uccelli, A., Moretta, L. and Pistoia, V. (2008) Mesenchymal stem cells in health and disease. Nature Reviews Immunology 8, 726-736

An exciting and multifaceted look at the biology of mesenchymal stem cells.

Slack, J.M. (2008) Origin of stem cells in organogenesis. Science 322, 1498-1501

A developmental biologist's thought-provoking, often contrarian, view of adult tissue regeneration.

\section{Website}

The public homepage of the Cardiovascular Cell Therapy Research Network provides background and information for several ongoing multicentre clinical trials in the USA using stem cells for cardiac therapy:

http://ccct.sph.uth.tmc.edu/cctrn/Public/PublicHome.aspx 


\section{Figure}

Figure 1. Putative paracrine effects of stem cells in ischaemic myocardium.

Table

Table 1. Characteristics of stem cell populations used for cardiac repair.

\section{Citation details for this article}

Steven J. Joggerst and Antonis K. Hatzopoulos (2009) Stem cell therapy for cardiac repair: benefits and barriers. Expert Rev. Mol. Med. Vol. 11, e20, July 2009, doi:10.1017/S1462399409001124 